\title{
ESTRUTURA DA VEGETAÇÃO LENHOSA EM DOIS FRAGMENTOS NATURAIS DE FLORESTAS INUNDÁVEIS (IMPUCAS) NO PARQUE ESTADUAL DO ARAGUAIA, MATO GROSSO ${ }^{1}$
}

Danielle Cristine de Figueiredo Barbosa², Beatriz Schwantes Marimon ${ }^{3}$, Eddie Lenza 3 , Ben Hur Marimon Junior $^{3}$, Edmar Almeida de Oliveira ${ }^{4}$ Leandro Maracahipes ${ }^{4}$

\begin{abstract}
RESUMO - As impucas são fragmentos florestais que sofrem inundação sazonal e se localizam em área de transição entre os biomas Cerrado e Floresta Amazônica. Este estudo objetivou avaliar e comparar a estrutura da vegetação de duas impucas com diferentes estados de conservação no Parque Estadual do Araguaia (PEA),

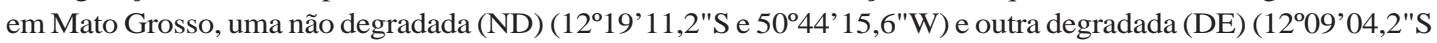
e 50 49'37"W). Foram demarcados cinco transectos subdivididos em 50 parcelas permanentes de $10 \times 20 \mathrm{~m}$. Todos os indivíduos lenhosos com diâmetro à altura do peito $\geq 5 \mathrm{~cm}$ foram amostrados. A impuca ND apresentou 28 famílias, 42 gêneros e 45 espécies, e a DE apresentou 22, 31 e 33, respectivamente. Na impuca ND, a densidade total foi de 2.177 ind.ha-1, a área basal de $35,23 \mathrm{~m}^{2}$.ha-1, e a diversidade de espécies de ShannonWiener de 2,87 nats.ind ${ }^{-1}$ e na DE foi 1.501 ind.ha ${ }^{-1}, 25,16 \mathrm{~m}^{2}$.ha- e 2,64 nats.ind ${ }^{-1}$, respectivamente. As espécies que mais se destacaram foram Licania apetala, Calophyllum brasiliense, Ochthocosmus multiflorus, Mabea paniculata, Tachigali froesii e Xylopia sp. A impuca DE apresentou menores riqueza, diversidade de espécies, densidade e dominância relativas do que a impuca ND, provavelmente devido ao histórico de perturbações e à inundação anual. A similaridade florística (Sørensen) entre ambas foi alta. Contudo, ao compará-las com impucas de Tocantins e outras florestas brasileiras, a similaridade foi baixa, sugerindo que as impucas de Mato Grosso e Tocantins apresentam identidade florística própria. Este estudo contribui para o conhecimento dos ecótonos brasileiros, para a elaboração do plano de manejo do PEA e para iniciativas de recuperação de impucas degradadas.
\end{abstract}

Palavras-chave: Fitossociologia, Transição Cerrado-Floresta Amazônica, Cerrado e Pantanal do Araguaia.

\section{STRUCTURE OF THE WOODY VEGETATION IN TWO NATURAL FRAGMENTS OF FLOODPLAIN FORESTS (IMPUCAS) IN THE ARAGUAIA STATE PARK, MATO GROSSO STATE}

\begin{abstract}
Impucas are seasonally inundated forest fragments located in the transition between the Cerrado and Amazonian Forest biomes. The objective of this study was to evaluate and to compare the vegetation structure of two impucas under different conservation status located at Araguaia State Park (AST), Mato Grosso State: non-altered vegetation structure (NA) (12 $19^{\prime} 11.2^{\prime \prime S}$ and 50 44'15.6"W) and altered vegetation

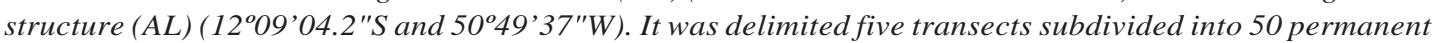
plots of $10 \times 20 \mathrm{~m}$. All woody individuals with diameter at breast height $\geq 5 \mathrm{~cm}$ were sampled. The NA impuca presented 28 families, 42 genera and 45 species and AL impuca 22 families, 31 genera and 33 species. Total density, basal area and Shannon-Wiener's diversity index were 2,177 ind.ha-1 $35.23 \mathrm{~m}^{2} . \mathrm{ha}^{-1}$ and 2.87 nats.ind ${ }^{-1}$
\end{abstract}

\footnotetext{
${ }^{1}$ Recebido em 19.08.2009 e aceito para publicação em 14.04.2011.

${ }^{2}$ Mestrado em Ciências Ambientais pela Universidade do Estado de Mato Grosso, UNEMAT, Brasil. E-mail: <danicris04@gmail.com>.

${ }^{3}$ Departamento de Ciências Biológicas, Universidade do Estado de Mato Grosso, Campus de Nova Xavantina, MT, Brasil. E-mail: <biamarimon@hotmail.com>, <eddielenza@yahoo.com.br>e <bhmjunior@gmail.com>.

${ }^{4}$ Programa de Pós-graduação em Ecologia e Conservação, Universidade do Estado de Mato Grosso, Campus de Nova Xavantina, MT, Brasil.E-mail: <edmardinei@hotmail.com>e <lmaracahipes@gmail.com>.
} 
in NA impuca and 1,501 ind.ha ${ }^{-1}, 25.16 \mathrm{~m}^{2} . \mathrm{ha}^{-1}$ and 2.63 nats.ind ${ }^{-1}$ in AL impuca, respectively. The most important species were Licania apetala, Calophyllum brasiliense, Ochthocosmus multiflorus, Mabea paniculata, Tachigali froesii and Xylopia sp. The AL impuca presented smaller floristic richness, species diversity, density and relative dominance in comparison with NA impuca, probably due the historic of disturbance and annual flooding. Floristic similarity (Sørensen) was high between ASP impucas. However, when they were compared with those of Tocantins and other Brazilian forests, the similarity was low, suggesting that impucas from Mato Grosso and Tocantins states presented their own floristic identity. This study contributes to the knowledge of the Brazilian ecotones, for elaboration of ASP management plan and for actions of recovery degraded impucas.

Keywords: Phytosociology, Cerrado-Amazon Forest transition, Cerrado and Araguaia River Floodplain.

\section{INTRODUÇÃO}

As impucas são florestas inundáveis que ocorrem na extensa planície de inundação do Araguaia, também conhecida por Pantanal do Araguaia, nos estados de Tocantins (MARTINS et al., 2002; 2006; 2008; BRITO et al., 2006; 2008) e Mato Grosso (MARIMON; LIMA, 2001; MARIMON et al., 2008). De acordo com Martins et al. (2002; 2006) e Brito et al. (2006), essas florestas ocorrem em depressões naturais do terreno e estão sujeitas a alagamento e a maior tempo de retenção de água em relação às outras fitofisionomias locais. Em função desse alagamento diferencial, as impucas ocorrem em forma de fragmentos naturais e isolados na paisagem, e apresentam especificidades florísticas, estruturais e fisionômicas que as diferenciam da vegetação do entorno (BRITO et al., 2006; 2008). O termo “impuca” é regional de Mato Grosso (EITEN, 1985) e a expressão “ipuca” é usada no estado de Tocantins (BRITO, 2005; MARTINS et al., 2002, 2006, 2008). Segundo Ferreira (2004), o termo origina-se do tupi e significa furo no igapó e água arrebentada.

Como esses fragmentos de floresta fazem a conexão entre rios, lagos e córregos no período de cheias, servindo também como ninhais para a avifauna, a sua degradação pode intensificar o processo migratório de predadores naturais e desencadear alterações no equilíbrio do ambiente (MARTINS et al., 2002). Tais conexões naturais fazem parte de um ciclo anual de cheias e vazantes que dirigem diversos processos ecológicos importantes ligados a esses ambientes inundáveis, como a reprodução e o crescimento de plantas, de peixes e invertebrados que estão relacionados às oscilações entre as fases aquática e terrestre dessas florestas (JUNK et al., 1989; SARMIENTO; PINILLOS, 2001).

As impucas estão localizadas na porção noroeste do bioma Cerrado, em uma região de tensão ecológica, na transição com a Floresta Amazônica, que foi classificada como de importância biológica extremamente alta (MARIMON;LIMA, 200; MARTINS et al., 2002; BRITO, 2005; MARIMON et al., 2006; 2006; 2008). Apesar das depressões do terreno que propiciam o alagamento no período das chuvas e da retenção de água por longos períodos (MARIMON et al., 2008), as impucas diferem das florestas de galeria, que, segundo Ribeiro e Walter (2008), acompanham rios de pequeno porte e córregos no Brasil central. A posição geográfica pré-amazônica das impucas pode fazer com que sofram influências florísticas dos dois biomas, caracterizando uma vegetação transicional com características próprias, mas ainda pouco conhecidas e estudadas (BRITO, 2005).

Tendo em vista a escassez de informações sobre impucas em Mato Grosso e o avanço de atividades antrópicas que colocam em risco a integridade dessas florestas, este estudo teve por objetivo avaliar e comparar a composição florística e a estrutura da vegetação de duas impucas com diferentes estados de conservação no Parque Estadual do Araguaia (PEA). Este estudo visa contribuir para a elaboração do plano de manejo do Parque e o desenvolvimento de iniciativas de recuperação de impucas degradadas.

\section{MATERIAL E MÉTODOS}

O estudo foi realizado em dois fragmentos naturais de florestas inundáveis (impucas) localizados no Parque Estadual do Araguaia (PEA), Mato Grosso. O PEA apresenta 223.619,54 hectares de área e localiza-se no Pantanal do Araguaia, na zona de transição entre os biomas Cerrado e Floresta Amazônica, em uma extensa planície sazonalmente inundada pelo Rio das Mortes e pelo Rio Araguaia (MARIMON et al., 2006; 2008).

APlanície do Araguaia é uma unidade geomorfológica de sedimentação, apresentando topografia plana e baixa altitude (180 a 230m), nível do lençol freático superficial 
elevado e predominância de Plintossolos e Gleissolos, ambos com impedimento de drenagem que favorece a inundação sazonal (BRASIL, 1994; MARTINS et al., 2002). O clima é do tipo Awi, segundo a classificação de Köppen (COCHRANE et al., 1985), com estação seca entre abril e setembro e chuvosa entre outubro e março. Entre os meses de dezembro e abril, o Parque Estadual do Araguaia permanece quase totalmente inundado, apresentando uma lâmina d'água com altura que pode variar de 0,2 m, em porções planas dos campos graminosos, a 2,5 m em depressões onde estão localizadas as florestas inundáveis (MARIMON;LIMA, 2001; MARIMON et al., 2008).

As impucas amostradas foram selecionadas de acordo com observações preliminares, visando estabelecer dois padrões distintos, um de vegetação degradada (DE) e um de vegetação não degradada (ND). A impuca DE (1209’04,2"S e 5049'37"W) apresentou elevada ocorrência de indivíduos de Scleria sp. (capimnavalha ou tiririca-de-vampira), marcas de fogo na maioria dos troncos das árvores, presença de clareiras, proximidade de áreas habitadas (sedes de fazendas) e estradas, e gado. A impuca ND (12 ${ }^{\circ} 19^{\prime} 11,2^{\prime \prime S ~ e ~}$ $50^{\circ} 44^{\prime} 15,6$ "W) apresentou uma situação antagônica em todos os aspectos anteriormente mencionados.

Não há atividades de cultivo de grãos nas proximidades das impucas estudadas no PEA. Nesse caso, os impactos sofridos na impuca DE estão diretamente relacionados ao acesso do gado bovino, que afeta a comunidade de plântulas (pisoteio) em um processo similar ao descrito por Vzzotto et al. (2000) e Santos et al. (2008), e ao fogo, que penetra na floresta no período da seca, causando danos principalmente à regeneração natural e à malha de raízes das árvores (NEPSTAD et al., 1999; MARTINS et al., 2002). Um dia após a conclusão da amostragem nesta impuca, em setembro de 2007, foi registrada uma queimada em seu interior, e a rápida expansão do fogo foi facilitada pela presença de Scleria sp. Nesta impuca também foram observadas árvores tombadas com marcas de queimada na base do tronco.

Em cada impuca, foi demarcado um hectare em transeções paralelas distantes 50 m entre si e com 20 m de largura, que transpuseram a floresta transversalmente de uma borda a outra. As transeções foram subdivididas em 50 parcelas permanentes de 10 x 20 m onde foram medidos o diâmetro e a altura e identificados todos os indivíduos com DAP (diâmetro ao nível do peito) $\geq 5 \mathrm{~cm}$, incluindo lianas, palmeiras e indivíduos mortos em pé.

Foi coletado material vegetativo e reprodutivo de todas as espécies amostradas, o qual foi herborizado, identificado e tombado no acervo do Herbário NX (UNEMAT-Campus de Nova Xavantina). O sistema de classificação utilizado foi o APG II (2003).

Para cada hectare, foram calculados os parâmetros fitossociológicos: densidade, frequência e dominância relativas e o valor de importância (MÜLLER-DOMBOIS; ELLENBERG, 1974), com o uso do programa Fitopac 1 (SHEPHERD, 1994). Também foram calculados o índice de diversidade de espécies de Shannon-Wiener ( $\mathrm{H}^{\prime}$; nats ind. ${ }^{-1}$ ) e a equabilidade de Pielou (J) (MAGURRAN, 1988). Os resultados do H' determinados para cada impuca foram comparados utilizando-se o teste $t$ de Hutcheson (ZAR, 1999).

Tendo em vista que na literatura científica só há estudos detalhados sobre duas impucas no estado de Tocantins (BRITO, 2005; BRITO et al., 2006; 2008; MARTINS et al., 2008), verificou-se a similaridade florística dessas com as deste estudo e com outras florestas associadas a corpos d’água (inundáveis ou não) dos biomas Cerrado e Floresta Amazônica. Para avaliar a similaridade florística, foi utilizado o índice de Sørensen (KENT; COKER, 1992).

Em cada impuca, foram coletadas 20 amostras de solos distribuídas ao acaso dentro das parcelas, entre 0 e $20 \mathrm{~cm}$ de profundidade, para a determinação dos parâmetros de fertilidade. As amostras foram submetidas à análise química no Laboratório Agroanálise em Cuiabá-MT, seguindo o que foi proposto por Embrapa (1979).

\section{RESULTADOS}

Os solos das impucas estudadas são distróficos, álicos, fortemente ácidos, com baixa saturação e soma de bases e elevadas concentrações de matéria orgânica, capacidade de troca catiônica e teores de N, P e K (Tabela 1).

Foram amostradas, nas duas impucas, 52 espécies, 48 gêneros e 31 famílias botânicas. Na impuca ND, foram registradas 45 espécies, 42 gêneros e 28 famílias, e na impuca DE foram 33 espécies, 31 gêneros e 22 famílias (Tabela 2). O índice de diversidade de espécies de Shannon-Wiener (H’) calculado para a impuca ND foi

Revista Árvore, Viçosa-MG, v.35, n.3, p.457-471, 2011 
Tabela 1 - Resultado das análises químicas dos solos de dois fragmentos de florestas inundáveis (impucas) no Parque Estadual do Araguaia, Mato Grosso. ND: floresta inundável não degradada (n=20); DE: floresta inundável degradada $(n=20)$. Médias e valores entre parênteses correspondem ao desvio-padrão (SB: soma de bases trocáveis; CTC: capacidade de troca catiônica; V: saturação por bases; e M.O.: matéria orgânica).

Table 1 - Results from chemical soil analyses for two floodplain forest fragment (impucas) in Araguaia State Park, Mato Grosso state, Brazil. ND: non-altered flooded forest $(n=20)$; DE: altered flooded forest $(n=20)$. Means and standard deviation in parenthesis (Parâmetros: parameters; SB: sum of exchangeable bases; V: base saturation; M. O.: organic matter).

\begin{tabular}{lcc}
\hline Parâmetros & ND & DE \\
\hline $\mathrm{pH} \mathrm{H} \mathrm{H}_{2} \mathrm{O}$ & $4,6(0,12)$ & $4,6(0,13)$ \\
$\mathrm{P}\left(\mathrm{mg}_{\mathrm{dm}}\right)$ & $25,4(13,2)$ & $23,5(11,7)$ \\
$\mathrm{K}^{+}\left(\mathrm{mg}^{-3} \mathrm{dm}^{-3}\right)$ & $122,8(36,1)$ & $110,0(31,5)$ \\
$\mathrm{Ca}^{++}\left(\mathrm{cmol}_{\mathrm{c}} \cdot \mathrm{dm}^{-3}\right)$ & $0,30(0,09)$ & $0,25(0,08)$ \\
$\mathrm{Mg}^{++}\left(\mathrm{cmol}_{\mathrm{c}} \cdot \mathrm{dm}^{-3}\right)$ & $0,22(0,07)$ & $0,17(0,07)$ \\
$\mathrm{Al}^{+++}\left(\mathrm{cmol}_{\mathrm{c}} \cdot \mathrm{dm}^{-3}\right)$ & $2,61(0,56)$ & $3,0(0,47)$ \\
$\mathrm{M} \cdot \mathrm{O} \cdot\left(\mathrm{gm}^{-3}\right)$ & $69,7(5,12)$ & $90,2(10,6)$ \\
$\mathrm{N}\left(\mathrm{g} \cdot \mathrm{kg}^{-1}\right)$ & $7,04(1,67)$ & $11,45(1,18)$ \\
$\mathrm{SB}\left(\mathrm{cmol}_{\mathrm{c}} \cdot \mathrm{dm}^{-3}\right)$ & $0,83(0,23)$ & $0,71(0,15)$ \\
$\mathrm{CTC}\left(\mathrm{cmol}_{\mathrm{c}} \cdot \mathrm{dm}^{-3}\right)$ & $16,1(1,85)$ & $19,7(2,2)$ \\
$\mathrm{V}(\%)$ & $5,2(1,2)$ & $3,6(0,7)$ \\
$\mathrm{Ca} / \mathrm{Mg}$ & $1: 1,36$ & $1: 1,47$ \\
\hline
\end{tabular}

de 2,87 nats.ind ${ }^{-1}$ e, para a impuca DE, foi 2,64, valores que diferiram significativamente entre si (teste $t$ de Hutcheson, $t=5,6, p<0,001)$. Por outro lado, a equabilidade de Pielou ( $\mathrm{J}$ ) foi similar entre as duas impucas ( $\mathrm{ND}=0,74$ e $\mathrm{DE}=0,75)$, ambas apresentando valores reduzidos, devido à elevada dominância numérica de poucas espécies. Na impuca ND, Micropholis gardneriana concentrou quase $30 \%$ de todos os indivíduos, enquanto $24 \%$ dos indivíduos amostrados na impuca DE pertenceram à Licania apetala (Tabela 2).

Na impuca ND foi registrada uma densidade total de 2.177 ind.ha-1, sendo 2.003 vivos e 174 mortos em pé (8\% do total). Na impuca DE, foi registrado um total de 1.501 ind.ha ${ }^{-1}$, sendo 1.336 vivos e 165 mortos (11\%). A área basal dos indivíduos vivos foi de 35,23 m².ha-1 na impuca ND e 25,16 $\mathrm{m}^{2}$. ha-1 na DE (Tabela 2).

As famílias que apresentaram maior VI (valor de importância) na impuca ND foram Sapotaceae (20,9\% do VI total), Chrysobalanaceae (12,1\%), Ebenaceae (7,8\%), Clusiaceae (5,6\%) e Ixonanthaceae (5,3\%), correspondendo a cerca de $52 \%$ do VI da comunidade (Tabela 2). Na impuca DE, Chrysobalanaceae (20,3\% do VI total), FabaceaeFaboideae (11,5\%), Fabaceae-Caesalpinioideae (11,3\%), Ixonanthaceae (11,2\%) e Euphorbiaceae (7,7\%) foram as mais importantes, correspondendo a $62 \%$ do VI total.

As espécies que apresentaram maiores densidades na impuca ND foram Micropholis gardneriana, Ochthocosmus multiflorus, Combretum laxum, Licania kunthiana e Roupala montana, somando 54,7\% do total de indivíduos amostrados e 40,6\% do VI total (Tabela 2). As maiores densidades da impuca DE foram registradas para Licania apetala, O. multiflorus, Tachigali froesii, Xylopia sp. e Mabea paniculata que representam 63,5\% do total de indivíduos e 54,1\% do VI total (Tabela 2). As espécies que apresentaram maiores áreas basais na impuca ND foram $M$. gardneriana, Calophyllum brasiliense, L. kunthiana, L. apetala e Diospyros guianensis, e na DE foram L. apetala, T. froesii, O. multiflorus e Leptolobium nitens (Tabela 2).

As dez espécies de maior valor de importância (VI) corresponderam a cerca de $63 \%$ do VI total na impuca ND e $77 \%$ na DE, indicando alta representatividade de poucas espécies nas comunidades amostradas (Tabela 2). Entre essas espécies, apenas Licania apetala, Ochthocosmus multiflorus e Calophyllum brasiliense estiveram entre as dez mais importantes nas duas impucas. Por outro lado, Micropholis gardneriana, Licania kunthiana, Combretum laxum, Diospyros guianensis, Roupala montana, Ternstroemia candolleana e Eschweilera ovata apresentaram os dez valores mais expressivos de VI apenas na impuca ND, enquanto Tachigali froesii, Leptolobium nitens, Xylopia sp., Mabea paniculata, Ormosia excelsa, Duroia prancei e Mauritiella armata estiverem entre as dez espécies melhor representadas em VI apenas na impuca DE (Tabela 2). 


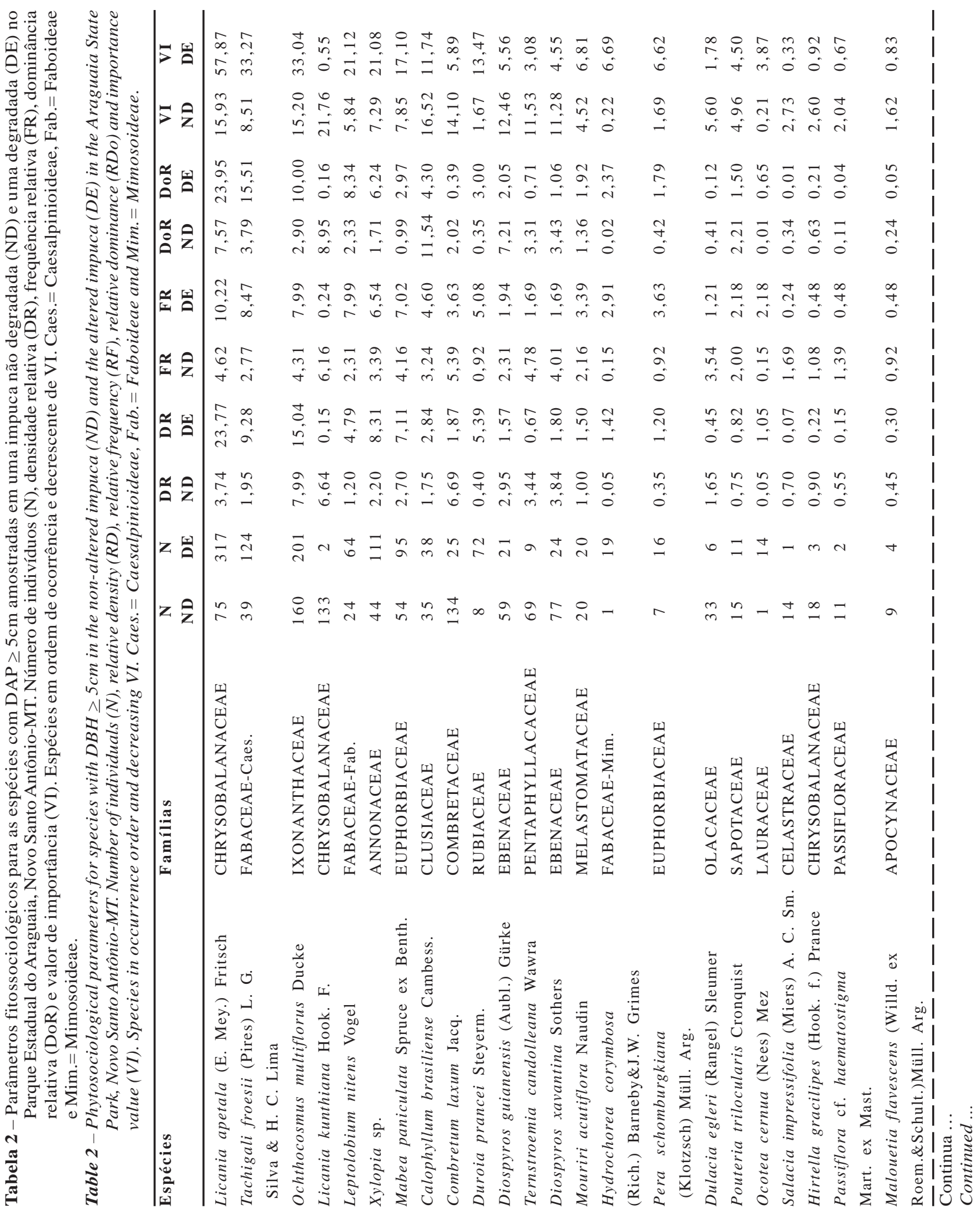




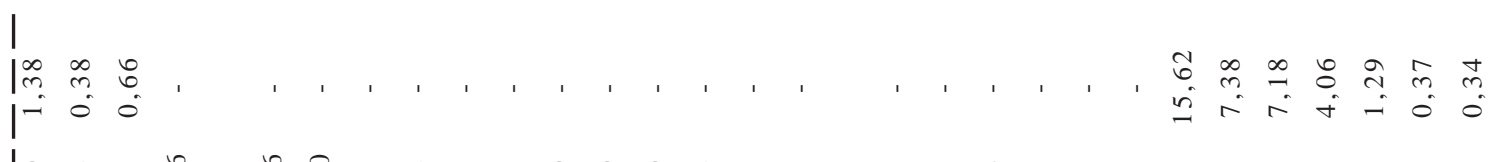

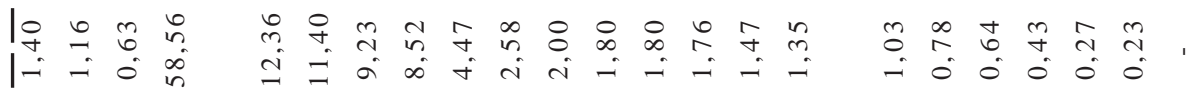

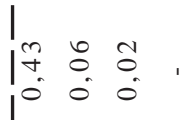

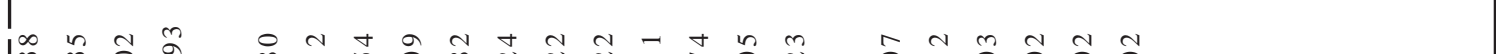

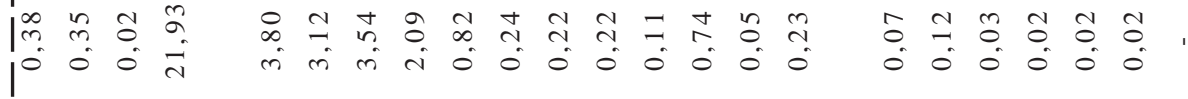

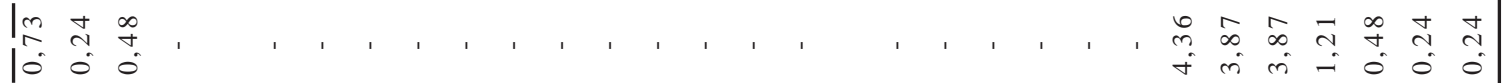

In

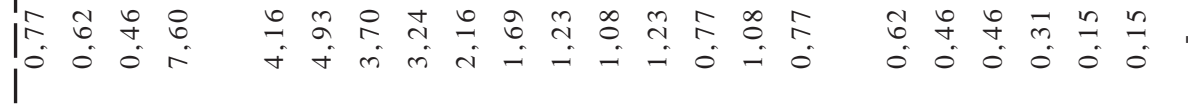

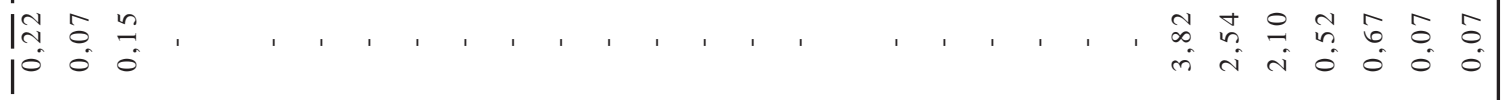

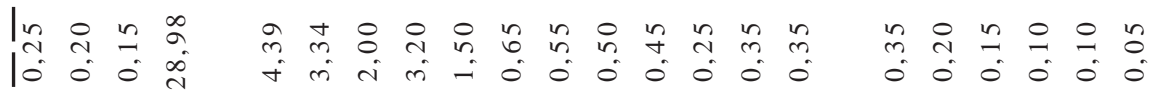

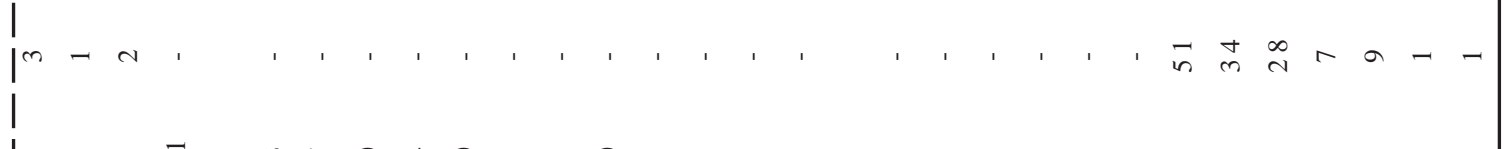

|

i

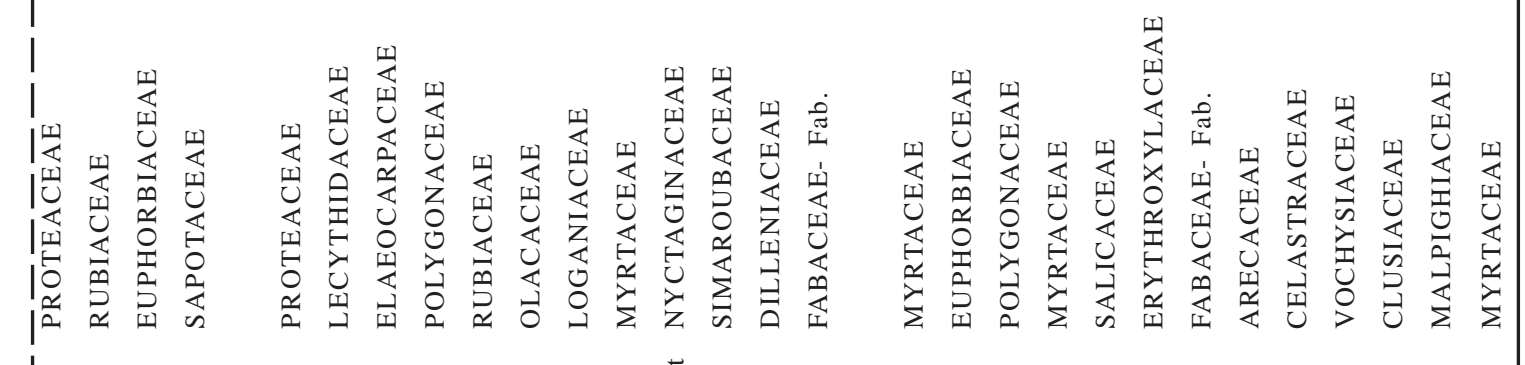

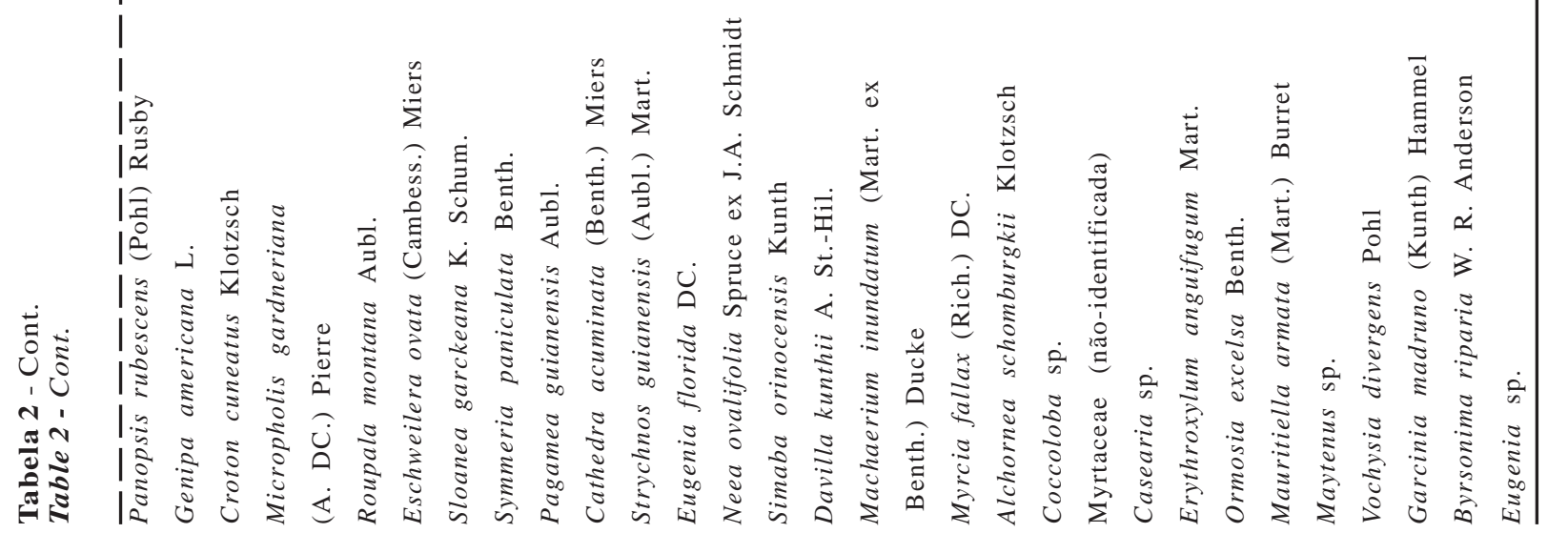

Revista Árvore, Viçosa-MG, v.35, n.3, p.457-471, 2011 
A similaridade florística entre as duas impucas deste estudo foi elevada (67\%). Das 52 espécies amostradas nas duas impucas do Parque Estadual do Araguaia (ND e DE), 26 foram comuns, 19 exclusivas da impuca ND e sete exclusivas da impuca DE (Tabela 2). Entretanto, apesar da elevada similaridade florística, a estrutura da vegetação foi distinta entre as impucas. A impuca DE apresentou menor riqueza e diversidade de espécies, menor densidade e menor área basal do que a ND (Tabela 2).

Comparando-se as impucas deste estudo com as de Tocantins (BRITO, 2005; BRITO et al., 2006; 2008; MARTINS et al., 2008), a similaridade florística entre as impucas não-degradadas (MT e TO) foi inferior a $15 \%$, e entre as impucas degradadas foi inferior a $4 \%$, valores considerados baixos. Em geral, as impucas deste estudo apresentaram maior similaridade florística com uma floresta inundável (21\%) e uma impuca (26\%) localizadas na Planície do Araguaia (RATTER, 1987; MARIMON; LIMA, 2001).

Comparações da composição florística das impucas deste estudo com outras florestas inundáveis, de brejo ou higrófilas, de várzea, ripárias ou de galeria e cambarazal (OLIVEIRA-FILHO, 1989; KLINGE et al., 1995; MARQUES et al., 2003; ALMEIDA et al., 2004; DAMASCENO-JÚNIOR et al., 2005; ARIEIRA; NUNES DA CUNHA, 2006) resultaram em valores do índice de Sørensen inferiores a $10 \%$.

\section{DISCUSSÃO}

Os parâmetros dos solos das impucas deste estudo foram similares aos registrados por Correia et al. (2001) e Reatto et al. (2001) em solos de florestas de galeria do Brasil central. Os reduzidos teores de Ca e Mg nas impucas do PEA (deste estudo) são similares aos registrados por Martins et al. (2006; 2008) em duas impucas no estado de Tocantins e por Ratter (1987) em florestas inundáveis na Ilha do Bananal. Os valores de $\mathrm{pH}$ registrados neste estudo estão de acordo com o que foi registrado por Martins et al. (2006), segundo os quais, valores entre 4,1 e 5,5 representam as condições gerais de acidez dos solos na Planície do Araguaia.

Os valores de $\mathrm{P}$ encontrados nas impucas do PEA (> $24 \mathrm{mg} . \mathrm{dm}^{-3}$ ) são muito superiores aos valores registrados em Tocantins ( $<3$ mg.dm ${ }^{-3}$ ) (RATTER, 1987; MARTINS et al., 2008), indicando que essas florestas inundáveis podem apresentar variação na disponibilidade deste elemento de uma região para outra, tal como observado por Haugaasen e Peres (2006), que encontraram maiores concentrações de $\mathrm{P}$ em solos de uma floresta de igapó, quando comparada a uma floresta de várzea na Amazônia. Alguns estudos demonstraram que a concentração de P pode aumentar quando os solos sofrem inundação, e a mobilização deste elemento provavelmente está associada à biogeoquímica de outros elementos (MOORE et al., 1992; CHACON et al., 2005). A relação Ca:Mg registrada nas impucas do PEA foi similar à de Tocantins, o que revela a natureza do material de origem desses solos (MARTINS et al., 2006).

Os elevados teores de Al e da acidez e a baixa concentração de Ca e bases trocáveis nos solos das impucas estudadas representam um padrão similar ao encontrado em florestas de várzea e igapó na Amazônia, que também estão sujeitas à inundação sazonal (FURCH; KLINGE, 1989; HAUGAASEN; PERES, 2006; FAJARDO et al., 2009). Neste estudo, os valores de Al são mais elevados do que os encontrados por Martins et al. (2008) nos solos de duas impucas de Tocantins $\left(<1,3 \mathrm{cmol}_{\mathrm{c}} \cdot \mathrm{dm}^{-3}\right)$. Tais condições edáficas das impucas, especialmente as do PEA, associadas ao encharcamento sazonal, pressupõem adaptações fisiológicas particulares das espécies para competir nesse ambiente extremo (SCARANO et al., 1997), especialmente na fase de estabelecimento das plântulas. Nesse caso, as elevadas concentrações de matéria orgânica resultantes da baixa decomposição no ambiente anaeróbico encharcado (CORREIA et al., 2004), ao lado dos altos teores de $\mathrm{N}$, P e K nos solos das impucas estudadas, podem ajudar na compensação dos fatores extremos. Infelizmente, além do trabalho de Tocantins, nenhum outro sobre as condições edáficas de impucas está disponível na literatura para mais comparações.

As duas impucas de Tocantins, estudadas por Martins et al. (2008) e Brito et al. (2006; 2008), apresentaram maior riqueza de famílias (35 na impuca antropizada e 34 na preservada), gêneros (57 e 44) e espécies (70 e 49) e maior diversidade de espécies ( $\mathrm{H}^{\prime}=3,44$ e 2,97 nats.ind ${ }^{-1}$ ) em relação às impucas deste estudo. Além disso, foi observada baixa similaridade florística entre as impucas do PEA e aquelas de Tocantins, bem como diversas espécies típicas de formações savânicas do Cerrado nestas últimas. Esses resultados sugerem que há uma diferenciação florística e estrutural entre as impucas de Mato Grosso e Tocantins, e novos estudos são necessários para conhecer as causas destas

Revista Árvore, Viçosa-MG, v.35, n.3, p.457-471, 2011 
diferenças, que podem estar associadas a diferentes regimes de inundação, estágios de sucessão, histórico de perturbação e estados de conservação (BARBOSA et al., 2010).

Os baixos valores de diversidade e riqueza de espécies das impucas do PEA em relação a outras florestas ripárias de Mato Grosso, estudadas por Marimon et al. (2002), Ivanauskas et al. (2004) e Kunz et al. (2008), podem ser atribuídos à característica de maior e mais prolongado alagamento das impucas, visto que as referidas florestas estão sujeitas a inundações sazonais de drenagem rápida, e as impucas estudadas podem permanecer inundadas por até cinco meses. Guarino e Walter (2005) observaram que florestas localizadas em áreas periodicamente inundáveis apresentam menor diversidade de espécies quando comparadas a florestas sobre solos bem drenados. Semelhante às impucas deste estudo, as matas de brejo no estado de São Paulo (TORRES et al., 1994; IVANAUSKAS et al., 1997; TONIATO et al., 1998) apresentaram baixos valores de diversidade devido ao alagamento como fator restritivo.

Apesar disso, a densidade total de indivíduos amostrados nas impucas do PEA pode ser considerada elevada, visto que os valores foram superiores àqueles encontrados em outras florestas inundáveis (KLINGE et al., 1995; IVANAUSKAS et al., 1997; DAMASCENOJUNIOR et al., 2005) ou em impuca preservada (BRITO et al., 2006; 2008). Nesse caso, apesar das impucas do PEA estarem sujeitas ao impacto de queimadas e do gado, sua localização em uma unidade de conservação pode ser fator determinante de maior número de indivíduos.

A área basal da impuca ND $\left(35,23 \mathrm{~m}^{2} \cdot\right.$ ha $\left.^{-1}\right)$ foi similar aos resultados registrados por Brito et al. (2008) na impuca preservada de Tocantins $\left(31,16 \mathrm{~m}^{2} . \mathrm{ha}^{-1}\right)$, por Pitman et al. (2001) em 24 áreas de floresta de terra firme na Amazônia ( $\left.\approx 30 \mathrm{~m}^{2} \cdot \mathrm{ha}^{-1}\right)$, além de estar dentro dos limites observados por Lamprecht (1972) para florestas da América do Sul (30-40 m².ha-1). O valor de área basal registrado na impuca ND permite que ela seja caracterizada como floresta tropical densa, de acordo com a classificação proposta por Brown e Lugo (1992) para florestas inundáveis, confirmando o bom estado de conservação desta impuca.

A impuca DE apresentou menor densidade, menor riqueza e diversidade de espécies e menor área basal, refletindo um padrão estrutural diferente da impuca
ND (Tabela 2). Com relação à área basal, a impuca DE $\left(25,16 \mathrm{~m}^{2} \cdot \mathrm{ha}^{-1}\right)$ pode ser classificada como floresta aberta, segundo Pires e Prance (1985). Entretanto, tal como observado por Kunz et al. (2008), os valores de área basal são diretamente influenciados pelo diâmetro mínimo da amostragem, o tipo florestal e o estado de conservação da área estudada. Assim, a menor área basal observada na impuca DE em relação à impuca ND pode ser explicada pelo maior grau de degradação da primeira, uma vez que a fitofisionomia e os critérios de amostragem foram os mesmos. Nesse caso, os impactos do fogo e da presença do gado podem estar contribuindo para a redução da densidade e da biomassa da vegetação lenhosa na impuca DE, promovendo maior luminosidade e alterando as características da comunidade, tal como observado por Martins et al. (2002) em impucas de Tocantins. Segundo Felfili et al. (2000) e Henriques e Hay (2002), o fogo pode ser responsável pela morte e exclusão de algumas espécies sensíveis e pela redução no número de indivíduos.

O percentual de indivíduos mortos em pé amostrados na impuca ND (8\%) foi semelhante àquele registrado por Brito et al. (2008) na impuca preservada de Tocantins (8,89\%). Felfili (2000) considera que o percentual de mortos em pé em florestas de galeria bem preservadas do Brasil central encontra-se em torno de 5\%. Nesse caso, para impucas, o percentual de mortos esperado pode ser moderadamente mais elevado do que aquele registrado em florestas de galeria, uma vez que as impucas se desenvolvem sobre substratos sujeitos a alagamentos, onde as taxas de mortalidade seriam naturalmente maiores.

A proporção de indivíduos mortos amostrados na impuca DE (11\%) do PEA foi superior àquela registrada na impuca antropizada de Tocantins (3,22\%) (BRITO, 2005; BRITO et al., 2008). Considerando que a impuca DE deste estudo e a antropizada, estudada em Tocantins encontram-se em diferentes condições ambientais e em diferentes níveis de impactos, novos estudos são necessários para avaliar as diferenças encontradas na proporção de indivíduos mortos nas duas áreas (TO e MT).

A elevada representatividade em VI, densidade e número de espécies da família Chrysobalanaceae nas impucas deste estudo, é uma importante característica florística dessas florestas. Esta família é considerada uma das principais em florestas de terra firme da Amazônia (PRANCE et al., 1976 apud KLINGE et al., 1995) e a 
sua abundância nos neotrópicos seria devido à habilidade de se adaptar a diferentes habitats (PRANCE, 1992). Além disso, é representada por espécies essencialmente amazônicas, que penetrariam no Cerrado através das florestas de galeria (PRADO;GIBBS, 1993) e demais formações florestais, como seria o caso das impucas.

As famílias Sapotaceae (PINTO; OLIVEIRA-FILHO, 1999) e Ixonanthaceae (BERRY; RAMÍREZ, 1995) também apresentariam vínculo florístico fortemente associado à Floresta Amazônica e, nesse caso, a elevada importância das referidas famílias nas impucas estudadas pode estar relacionada à localização pré-amazônica do PEA, confirmando a forte influência daquele bioma na flora das impucas. Por outro lado, Euphorbiaceae é uma família comum em florestas de galeria do Brasil central (SILVAJÚNIOR et al., 2001), evidenciando também uma ligação florística com formações florestais do Cerrado e o caráter ecotonal das impucas do PEA.

Comparando-se as impucas do PEA com as de Tocantins, observou-se que a família Sapotaceae, que foi a mais importante na impuca ND deste estudo, e Ixonanthaceae, que foi importante nas duas impucas de Mato Grosso, não foram mencionadas nos estudos de Tocantins (BRITO, 2005; BRITO et al., 2008; MARTINS et al., 2008). Este aspecto sugere que a composição florística das impucas pode variar dependendo da localização geográfica, reforçando ainda mais a necessidade de ampliar os estudos em diferentes regiões do Brasil para garantir ampla caracterização desta fitofisionomia e o estabelecimento de estratégias de conservação.

As espécies Licania apetala, Ochthocosmus multiflorus, Mabea paniculata, Xylopia sp., Tachigali froesii e Calophyllum brasiliense podem ser consideradas típicas das impucas do PEA, pois apresentaram densidades superiores a 30 indivíduos em ambas e estiveram entre as dez de maior VI em pelo menos uma das impucas estudadas. Além de $C$. brasiliense e $T$. froesii (=Sclerolobium froesii), Leptolobium nitens (=Acosmium nitens), também foi considerada espécie comum de impucas em estudos florísticos pioneiros realizados por Eiten (1985) e Ratter (1987) na região. Entretanto, os moradores do PEA mencionam que ainda há muita extração de madeira das impucas, e as espécies mais procuradas são $C$. brasiliense (construção de casas e galpões) e Tachigali froesii (cercas e currais).
As sementes de C. brasiliense, apesar de não germinarem enquanto inundadas, germinam mesmo após vários meses de submersão e suas plântulas conseguem crescer normalmente tanto em solos inundados quanto em solos bem drenados (MARQUES; JOLY, 2000). Essas características fazem com que a espécie seja muito frequente em vários ambientes ribeirinhos do Brasil e em outros ambientes neotropicais, onde o solo é permanente ou periodicamente inundado (SCARANO et al., 1997), o que explica sua representatividade nas impucas estudadas.

A espécie Licania apetala também foi registrada entre as dez de maior densidade na impuca preservada de Tocantins (BRITO et al., 2008) e entre as mais importantes na estrutura de florestas de galeria do Brasil central (SILVA-JÚNIOR et al., 1996; 2001; SCHIAVINI et al., 2001). Ribeiro et al. (1999) observaram ainda que esta espécie apresenta ampla distribuição na Floresta Amazônica. Nesse caso, $L$. apetala parece ser uma das espécies com mais ampla área de distribuição em florestas inundáveis dos biomas Cerrado e Floresta Amazônica, provavelmente devido à sua tolerância ao encharcamento sazonal (SILVAJÚNIOR et al., 1996).

Considerando-se que a densidade de Licania kunthiana foi muito superior na impuca ND deste estudo, esta espécie pode não ser capaz de tolerar perturbações (fogo e pisoteio de gado) e por esse motivo, pode se adaptar melhor em ambientes não degradados. De acordo com Ratter et al. (1973), L. kunthiana é uma espécie característica de florestas sempre-verdes, que ocasionalmente estão sujeitas às inundações. Estudos para avaliar o crescimento de espécies de impucas deveriam incluir esta para testes.

As espécies Micropholis gardneriana, Licania kunthiana, Combretum laxum, Diospyros guianensis, Roupala montana, Ternstroemia candolleana e Eschweilera ovata podem ser consideradas indicadoras de impucas não degradadas, pois apresentaram alta densidade e importância estrutural (elevado VI) somente na impuca ND. Provavelmente essas espécies são menos resistentes ao fogo e ao pisoteio do gado. Apesar de M. gardneriana ter sido citada por Mendonça et al. (2008) como de ocorrência em florestas ciliares e de galeria do bioma Cerrado, este estudo representa o primeiro registro desta espécie como a de maior importância ecológica em uma floresta inundável. Entretanto, a

Revista Árvore, Viçosa-MG, v.35, n.3, p.457-471, 2011 
degradação ambiental das impucas do PEA poderá colocá-la em risco, uma vez que não houve registro dela na impuca DE.

Por outro lado, as espécies Licania apetala, Tachigali froesii, Leptolobium nitens, Xylopia sp., Mabea paniculata, Ormosia excelsa, Duroia prancei e Mauritiella armata foram mais abundantes e importantes na impuca DE, sendo consideradas indicadoras de impucas degradadas. As referidas espécies foram consideradas por Ratter (1987), Kubitzki (1989), Pott e Pott (1994), Varela et al. (2005) e Marimon e Lima (2001) invasoras ou comuns em florestas antropizadas do Pantanal e da Amazônia, ou na transição entre os biomas Cerrado e Floresta Amazônica. L. apetala e $T$. froesii, apesar de ocorrerem também na impuca $\mathrm{ND}$, quando registradas em elevadas densidades podem indicar impucas degradadas, uma vez que a abundância e o VI dessas espécies foram três vezes superiores na impuca DE. Nesse caso, os distúrbios provocados pelo fogo e pelo gado no PEA podem estar contribuindo para alterar a composição florística e a estrutura da vegetação das impucas, desfavorecendo um conjunto de espécies e favorecendo outro.

Dentre as 10 espécies de maior VI amostradas nas impucas do PEA, apenas quatro, Calophyllum brasiliense, Licania apetala, Diospyros guianensis e Eschweilera ovata também apareceram em destaque nas duas impucas de Tocantins (BRITO et al., 2006; 2008; MARTINS et al., 2008). Apesar das impucas de Mato Grosso e de Tocantins estarem localizadas na mesma planície de inundação, distantes entre si menos de 200 km em linha reta, apresentam composição florística e estrutura da vegetação diferenciadas, caracterizando que esta fitofisionomia pode variar dependendo do grau de interferência antrópica, nível de inundação, fatores edáficos, localização geográfica, dentre outros.

Um aspecto que precisa ser salientado é que as impucas estudadas em Tocantins encontram-se posicionadas geograficamente mais na borda da planície de inundação do Rio Araguaia do que as impucas estudadas em Mato Grosso. Este fato pode explicar a ocorrência de um grande número de espécies típicas de Cerrado sentido restrito nas impucas de Tocantins, como Curatella americana, Qualea grandiflora e Q. multiflora (BRITO et al., 2008; MARTINS et al., 2008), provavelmente refletindo menor grau de inundação e maior influência florística das fitofisionomias savânicas do bioma Cerrado. Assim, a divergência florística e estrutural observada entre as impucas do PEA e as de Tocantins pode ser determinada, em parte, pela influência da flora adjacente, ou seja, maior participação da flora amazônica nas impucas de Mato Grosso e maior participação da flora do Cerrado nas impucas de Tocantins.

As impucas do PEA apresentaram maior similaridade florística com as florestas inundáveis da Planície do Araguaia (RATTER, 1987; MARIMON;LIMA, 2001) provavelmente por estarem geograficamente mais próximas dessas do que das demais florestas comparadas. Neste caso, fatores como altitude, latitude e características climáticas podem ser determinantes para definir a similaridade florística entre florestas sazonalmente inundáveis dos biomas Cerrado e Floresta Amazônica. Estudos mais detalhados e levantamentos em diferentes áreas são imprescindíveis para compreender os padrões florísticos e fitogeográficos dessas importantes e raras formações vegetais.

Analisando-se as listagens da flora fanerogâmica compiladas por Ribeiro et al. (1999), Felfili et al. (2001) e Mendonça et al. (2008), observa-se que 60\% das espécies amostradas nas impucas do PEA foram registradas em diferentes fitofisionomias do Cerrado e 27\% também na Floresta Amazônica, confirmando a influência desses dois biomas na composição florística das impucas do PEA, localizadas em área de transição. Entre as espécies amostradas no PEA, 27\% também foram observadas em estudo efetuado por Pott e Pott (1994) no Pantanal mato-grossense (BARBOSA et al., 2010), sugerindo que a natureza alagável desses dois pantanais seleciona espécies tolerantes ao encharcamento sazonal. No entanto, a despeito da influência florística desses três ricos e diversos biomas brasileiros, as impucas apresentaram, em geral, baixa riqueza e diversidade da flora lenhosa, devido à seleção de poucas espécies adaptadas ao encharcamento sazonal.

\section{CONCLUSÃO}

A posição geográfica das impucas do Parque Estadual do Araguaia pode estar contribuindo para que sofram influências florísticas dos biomas Cerrado e Floresta Amazônica, representando uma vegetação transicional com características próprias. As espécies Licania apetala, Calophyllum brasiliense, Ochthocosmus multiflorus, Mabea paniculata, Tachigali froesii e Xylopia sp. podem ser consideradas típicas de impucas do Parque Estadual do Araguaia. 
A menor riqueza, diversidade de espécies, densidade e dominância relativa da impuca DE em relação à ND deve-se provavelmente ao histórico de perturbações sofrido por cada uma e ao período que estão sujeitas à inundação anual. Baseando-se na área basal por hectare, a impuca ND pode ser considerada uma floresta densa, e a impuca DE uma floresta aberta.

Os fatores responsáveis pela reduzida similaridade florística observada entre as impucas deste estudo e as impucas de Tocantins precisam ser avaliados em novos estudos. Entretanto, a reduzida similaridade pode estar relacionada às diferenças no regime de inundação, no histórico, no tipo de perturbação e na localização das impucas de Tocantins mais à margem da Planície do Araguaia, sugerindo maior influência das fitofisionomias savânicas do bioma Cerrado. É possível que as impucas de Mato Grosso e Tocantins apresentem identidade florística própria.

Os solos das impucas deste estudo apresentam características compatíveis com outras florestas sujeitas à inundação nos biomas Cerrado e Floresta Amazônica. Tais condições sugerem que este pode ser considerado um ambiente extremo não só pelo encharcamento sazonal e anoxia, mas também por condições edáficas que podem estar atuando na seleção de espécies arbóreas.

Apesar de se tratar de uma unidade de conservação, o histórico de perturbações antrópicas, provocadas pelo fogo e pelo gado no PEA, pode estar reduzindo a densidade, a riqueza e a diversidade florística e alterando a composição de espécies e a estrutura da vegetação de impucas. Dessa forma, este estudo é fundamental para embasar o plano de manejo do Parque Estadual do Araguaia, norteando estratégias de controle dos impactos e a escolha de espécies para recuperação de impucas já degradadas, reduzindo os riscos de extinção local e de alterações irreversíveis neste frágil e pouco conhecido ecossistema do estado de Mato Grosso.

\section{AGRADECIMENTOS}

À CAPES, pela bolsa concedida ao primeiro autor; à Fundação de Amparo à Pesquisa do Estado de Mato Grosso (FAPEMAT, Proc. 0650/2006), pelo financiamento da pesquisa; e à Secretaria do Estado de Meio Ambiente de Mato Grosso (SEMA-MT), por autorizar o desenvolvimento deste estudo e disponibilizar o uso da sede do Parque Estadual do Araguaia para o alojamento dos pesquisadores.

\section{REFERÊNCIAS}

ALMEIDA, S. S.; AMARAL, D. D.; SILVA, A. S. L. Análise florística e estrutura de Florestas de Várzea no estuário amazônico. Acta

Amazonica, v.34, n.4, p.513-524, 2004. APG II Angiosperm Phylogeny Group II. An update of the Angiosperm Phylogeny Group classification for the orders and families of flowering plants: APG II. Botanical Journal of the

Linnean Society, v.141, p.399-436, 2003.

ARIEIRA, J.; NUNES DA CUNHA, C.

Fitossociologia de uma floresta inundável monodominante de Vochysia divergens Pohl (Vochysiaceae), no Pantanal Norte, MT, Brasil. Acta Botanica Brasilica, v.20, n.3, p.569-580, 2006.

BARBOSA, D. C. F. et al. Composição florística de dois fragmentos naturais de florestas inundáveis (impucas) no Parque Estadual do Araguaia-MT. In: SANTOS, J. E.; GALBIATI, C.; MOSCHINI, L. E. (Orgs.). Gestão e educação ambiental - Água, biodiversidade e cultura. São Carlos: Rima, 2010. v.2. p.69-81.

BERRY, P. E.; RAMÍREZ, N. Taxonomic changes in Ixonanthaceae from the Venezuelan Guyana.

Novon, v.5, p.227-229, 1995.

BRASIL. Ministério do Meio Ambiente e da Amazônia Legal. IBAMA. Plano de ação emergencial para o Parque Nacional do Araguaia. Documento de Informações Básicas. 1994. 56p.

BRITO, E. R. Florística e estrutura de
fragmentos naturais de florestas
inundáveis - Ipucas - e identificação
de áreas degradadas da Fazenda Lago
Verde, Lagoa da Confusão-TO. 2005. $80 \mathrm{f}$.
Tese (Doutorado em Ciência Florestal) -
Universidade Federal de Viçosa, Viçosa, MG, 2005.

BRITO, E. R. et al. Estrutura fitossociológica de um fragmento natural de floresta inundável em área de orizicultura irrigada, município de Lagoa da Confusão, Tocantins. Revista Árvore, v.30, n.5, p.829-836, 2006.

Revista Árvore, Viçosa-MG, v.35, n.3, p.457-471, 2011 
BRITO, E. R. et al. Estrutura fitossociológica de um fragmento natural de floresta inundável em área de Campo Sujo, Lagoa da Confusão, Tocantins. Acta Amazonica, v.38, n.3, p.379386, 2008.

BROWN, S.; LUGO, A. E. Aboveground biomass estimates for tropical moist forests for Brazilian Amazon. Interciencia, v.17, n.1, p.8-18, 1992.

CHACON, N. et al. Implications of soil organic carbon at the biogeochemistry of iron and aluminum on soil phosphorus distribution in flooded forests of the lower Orinoco River, Venezuela. Biogeochemistry, v.73, p.555-556, 2005

COCHRAne, T. T. et al. Land in Tropical America. Cali: CIAT/Embrapa-CPAC, 1985.

CORREIA, J. R. et al. Influência de fatores edáficos na distribuição de espécies arbóreas em matas de galeria na região do Cerrado: uma revisão. In: RIBEIRO, J.F.; FONSECA, C. E. L.; SOUSA-SILVA, J. C. (Eds.). Cerrado: caracterização e recuperação de matas de galeria. Planaltina: Embrapa Cerrados, 2001. p.51-76.

CORREIA, J. R.; REATTO, A.; SPERA, S. T. Solos e suas relações com o uso e manejo. In: SOUZA, D. M. G.; LOBATO, E. (Eds.). Cerrado: correção do solo e adubação. Brasília: Embrapa Cerrados, 2004. p.29-61.

DAMASCENO-JUNIOR, G. A. et al. Structure, distribution of species and inundation in a riparian forest of Rio Paraguai, Pantanal, Brazil. Flora, v.200, p.119-135, 2005.

EITEN, G. Vegetation near Santa Teresinha, NE Mato Grosso. Acta Amazonica, v.15, n.3-4, p.275-301, 1985.

\section{EMPRESA BRASILEIRA DE PESQUISA} AGROPECUÁRIA - EMBRAPA. Centro Nacional de Pesquisa de Solos. Manual de métodos de análises de solo. 2.ed. Rio de Janeiro: 1979. 212p.

FAJARDO, J. D. V.; SOUZA, L. A. G.; ALFAIA, S. S. Características químicas de solos de várzeas sob diferentes sistemas de uso da terra, na calha dos rios baixo Solimões e médio Amazonas. Acta Amazonica, v.39, n.4, p.731-740, 2009.
FELFILI, J. M. et al. Changes in the floristic composition of cerrado sensu stricto in Brazil over a nine-year period. Journal of Tropical Ecology, v.16, p.579-590, 2000.

FELFILI, J. M. et al. Flora fanerogâmica das Matas de Galeria e Ciliares do Brasil Central. In: RIBEIRO, J. F.; FONSECA, C. E. L.; SOUSASILVA, J. C. (Eds.). Cerrado: caracterização e recuperação de Matas de Galeria. Planaltina: Embrapa, 2001. p.195-263.

FELFILI, J. M. Crescimento, recrutamento e mortalidade nas matas de galeria do Planalto Central. In: CAVALCANTI, T. T.; WALTER, B. M. T. (Orgs.). Tópicos atuais em botânica. LOCAL: SBB/Embrapa, 2000. p.152-158.

FERREIRA, A. B. H. Novo dicionário eletrônico aurélio da língua portuguesa. 3.ed. 2004. CD ROM.

FURCH, K.; KLINGE, H. Chemical relationship between vegetation, soil and water in contrasting inundation areas of Amazonia. In: PROCTOR, J. (Ed.). Mineral nutrients in tropical forest and savanna ecosystems. Oxford: Blackwell, 1989. p.189-204.

GUARINO, E. S. G.; WALTER, B. M. T. Fitossociologia de dois trechos inundáveis de Matas de Galeria no Distrito Federal, Brasil. Acta Botanica Brasilica, v.19, n.3, p.431-442, 2005.

HAUGAASEN, T.; PERES, C. A. Floristic, edaphic and structural characteristics of flooded and unflooded forests in the lower Rio Purús region of central Amazonia, Brazil. Acta Amazonica, v.36, n.1, p.25-36, 2006.

HENRIQUES, R. P. B.; HAY, J. D. Patterns and dynamics of plant populations. In: OLIVEIRA, P. S.; MARQUIS, R. J. (Eds.). The cerrados of Brazil. New York: Columbia University Press, 2002. p.140-158.

IVANAUSKAS, N. M.; MONTEIRO, R.; RODRIGUES, R. R. Estrutura de um trecho de floresta Amazônica na bacia do alto rio Xingu. Acta Amazonica, v.34, n.2, p.275-299, 2004. 
IVANAUSKAS, N. M.; RODRIGUES, R. R.; NAVE, A. G. Aspectos ecológicos de um trecho de floresta de brejo em Itatinga, SP: florística, fitossociologia e seletividade de espécies.

Revista Brasileira de Botânica, v.20, n.2, p.139-153, 1997.

JUNK, W. J.; BAYLEY, P. B.; SPARKS, R. E. The flood pulse concept in river-floodplain systems. In: DODGE D. P. (Ed.). INTERNATIONAL LARGE RIVER SYMPOSIUM, 1989, Ottawa.

Proceedings... Ottawa: Canadian Special Publication of Fisheries and Aquatic Sciences, 1989. p.110-127.

KENT, M.; COKER, P. Vegetation description and analysis; a practical approach. London: Belhaven Press, 1992. 363p.

KLINGE, H.; ADIS, J.; WORBES, M. The vegetation of a seasonal várzea forest in the lower Solimões River, Brazilian Amazonia. Acta Amazonica, v.25, n.3-4, p.201-220, 1995.

KUBITZKI, K. The ecogeographical differentiation of Amazonian inundation forests. Plant Systematics and Evolution, v.162, p.285-304, 1989.

KUNZ, S. H. et al. Aspectos florísticos e fitossociológicos de um trecho de Floresta Estacional Perenifólia na Fazenda Trairão, Bacia do rio das Pacas, Querência-MT. Acta

Amazonica, v.38, n.2, p.245-254, 2008.

LAMPRECHT, H. Einige strukturmerkmale naturlicher Tropenwaldtypen und ihren waldbauliche bedeutung.

Forstwissenschaften Zentralblatt, v.91, p.270-277, 1972.

MAGURRAN, A. E. Ecological diversity and its measurement. London: Croom Helm, 1988. 179p.

MARIMON, B. S. et al. Observations on the vegetation of northeastern Mato Grosso, Brazil. IV. An analysis of the Cerrado-Amazonian Forest ecotone. Edinburgh Journal of Botany, v.63, n.2/3, p.323-341, 2006.

MARIMON, B. S. et al. Pantanal do Araguaia - ambiente e povo: guia de ecoturismo. Cáceres: UNEMAT, 2008. 95p.
MARIMON, B. S.; LIMA, E. S. Caracterização fitofisionômica e levantamento florístico preliminar no Pantanal dos Rios Mortes-Araguaia, Cocalinho, Mato Grosso, Brasil. Acta Botanica Brasilica, v.15, n.2, p.213-229, 2001.

MARIMON, B. S.; FELFILI, J. M.; LIMA, E. S. Floristics and phytosociology of the gallery forest of the Bacaba Stream, Nova Xavantina, Mato Grosso, Brazil. Edinburgh Journal of Botany, v.59, n.2, p.303-318, 2002.

MARQUES, M. C. M.; JOLY, C. A. Estrutura e dinâmica de uma população de Calophyllum brasiliense Camb. em floresta higrófila do sudeste do Brasil. Revista Brasileira de Botânica, v.23, n.1, p.107-112, 2000.

MARQUES, M. C. M.; SILVA, S. M.; SALINO, A. Florística e estrutura do componente arbustivoarbóreo de uma floresta higrófila da Bacia do Rio Jacaré-Pepira, SP, Brasil. Acta Botanica Brasilica, v.17, n.4, p.495-506, 2003.

MARTINS, A. K. E. et al. Relações SoloGeoambiente em áreas de ocorrência de ipucas na planície do Médio Araguaia - Estado de Tocantins. Revista Árvore, v.30, n.2, p.297310, 2006.

MARTINS, I. C. M. et al. Diagnóstico ambiental no contexto da paisagem de fragmentos florestais naturais “ipucas” no município de Lagoa da Confusão, Tocantins. Revista Árvore, v.26, n.3, p.299-309, 2002.

MARTINS, S. V. et al. Floristic composition of two wetland forests in Araguaian Plain, State of Tocantins, Brazil, and comparison with other areas. Revista Árvore, v.32, n.1, p.129-141, 2008.

MENDONÇA, R. C. et al. Flora vascular do Bioma Cerrado; checklist com 12.356 espécies. In: SANO, S. M.; ALMEIDA, S. P.; RIBEIRO, J. F.

Cerrado: ecologia e flora. Brasília: Embrapa Informação Tecnológica, 2008. v.2. p.422-1279.

MOORE, P. A.; REDDY, K. R.; GRAETZ, D. A. Nutrient transformations in sediments as influenced by oxygen supply. Journal of Environmental Quality, v.21, p.387-393, 1992.

Revista Árvore, Viçosa-MG, v.35, n.3, p.457-471, 2011 
MÜLlER-DOMBOIS, D.; ELLENBERG, H. Aims and methods of vegetation ecology. New York: J. Wiley \& Sons, 1974. 574p.

NEPSTAD, D. C.; MOREIRA, A. G.; ALENCAR, A. A. Floresta em chamas; origens, impactos e prevenção do fogo na Amazônia. Brasília: IPAM/ Woods Hole Research Center, 1999. 202p.

OLIVEIRA-FILHO, A. T. Composição florística e estrutura comunitária da floresta de galeria do Córrego da Paciência, Cuiabá (MT). Acta

Botanica Brasilica, v.3, n.1, p.91-112, 1989.

PINTO, J. R. R.; OLIVEIRA-FILHO, A. T. Perfil florístico e estrutura da comunidade arbórea de uma floresta de vale no Parque Nacional da Chapada dos Guimarães, MT, Brasil. Revista Brasileira de Botânica, v.22, n.1, p.53-67, 1999.

PIRES, J. M.; PRANCE, G. T. The vegetation types of the Brazilian Amazon. In: PRANCE, G. T.; LOVEJOY, T. E. (Ed.). Key

Environments: Amazonia. Oxford: Pergamon Press, 1985. p.109-145.

PITMAN, N. C. A. et al. Dominance and distribution of tree species in upper Amazonian terra firme forests. Ecology, v.82, n.8, p.2101-2117, 2001.

POTT, A.; POTT, V. J. Plantas do Pantanal. Corumbá: Embrapa-SPI, 1994. 320p.

PRADO, D. E.; GIBBS, P. E. Patterns of species distributions in the dry seasonal forests of South America. Annals of the Missouri

Botanical Garden, v.80, n.4, p.902-927, 1993.

PRANCE, G. T. The phytogeography of savanna species of neotropical Chrysobalanaceae. In: FURLEY, P. A.; PROCTOR, J.; RATTER, J. A. Nature and dynamics of forestSavanna Boundaries. London: Chapman \& Hall, 1992. p.295-330.

RATTER, J. A. et al. Observations on the vegetation of northeastern Mato Grosso I. The woody vegetation types of the XavantinaCachimbo Expedition area. Philosophical Transactions of the Royal Society of London, v.266, p.449-492, 1973.

Revista Árvore, Viçosa-MG, v.35, n.3, p.457-471, 2011
RATTER, J. A. Notes on the vegetation of the Parque Nacional do Araguaia (Brazil). Notes RBG Edinburgh, v.44, n.2, p.311-342, 1987.

REATTO, A. et al. Solos de ocorrência em duas áreas sob matas de galeria no Distrito Federal: aspectos pedológicos, uma abordagem química e físico-hídrica. In: RIBEIRO, J.F.; FONSECA, C. E. L.; SOUSA-SILVA, J. C. (Ed.). Cerrado: caracterização e recuperação de matas de galeria. Planaltina: Embrapa Cerrados, 2001. p.115-140.

RIBEIRO, J. E. L. S. et al. Flora da Reserva Ducke: Guia de identificação das plantas vasculares de uma floresta de terra-firme na Amazônia Central. Manaus: INPA, 1999. 816p.

RIBEIRO, J. F.; WALTER, B. M. T. As principais fitofisionomias do Bioma Cerrado. In: SANO, S. M.; ALMEIDA, S. P.; RIBEIRO, J. F. Cerrado: ecologia e flora. Brasília: Embrapa Informação Tecnológica, 2008. p.151-212.

SCARANO, F. R. et al. Plant establishment on flooded and unflooded patches of a freshwater swamp forest in southeastern Brazil. Journal of Tropical Ecology, v.14, p.793-803, 1997.

SCHIAVINI, I.; RESENDE, J. C. F.; AQUINO, F. G. Dinâmica de populações de espécies arbóreas em Mata de Galeria e Mata Mesófila na margem do Ribeirão Panga, MG. In: RIBEIRO, J. F.; FONSECA, C. E. L.; SOUSA-SILVA, J. C. (Ed.) Cerrado: caracterização e recuperação de Matas de Galeria. Planaltina: Embrapa Cerrados, 2001. p.267-299.

SHEPHERD, G. J. FITOPAC 1 - Manual do usuário. Campinas: UNICAMP, Departamento de Botânica, 1994. 88p.

SANTOS, A. L. S.; PEREIRA, E. C. G.; ANDRADE, L. H. C. Fragmentação florestal decorrente do uso do solo e do processo de degradação ambiental, no município de Junqueiro (AL). Caminhos de Geografia, v.9, n.25, p.115-120, 2008.

SARMIENTO, G.; PINILLOS, M. Patterns and processes in a Seasonally Flooded Tropical Plain: the Apure Llanos, Venezuela. Journal of Biogeography, v.28, n.8, p.985-996, 2001. 
SILVA-JÚNIOR, M. C. et al. Análise da flora de Matas de Galeria no Distrito Federal: 21 levantamentos. In: RIBEIRO, J. F.; FONSECA, C. E. L.; SOUSA-SILVA, J. C. (Ed.). Cerrado: caracterização e recuperação de Matas de Galeria. Planaltina: Embrapa, 2001. p.143-191.

SILVA-JÚNIOR, M. C.; FURLEY, P. A.; RATTER, J. A. Variations in tree communities and soils with slope in gallery forest, Federal District, Brazil. Advances in Hillslope Processes, v.1, p.451-469, 1996.

TONIATO, M. T. Z.; LEITÃO-FILHO, H. F.; RODRIGUES, R. R. Fitossociologia de um remanescente de floresta higrófila (mata de brejo) em Campinas, SP. Revista Brasileira de Botânica, v.21, n.2, p.197-210, 1998.
TORRES, R. B.; MATTHES, L. A. F.; RODRIGUES, R. R. Florística e estrutura do componente arbóreo de mata de brejo em Campinas, SP. Revista Brasileira de Botânica, v.17, n.2, p.189-194, 1994.

VARELA, V. P.; COSTA, S. S.; RAMOS, M. B. P. Influência da temperatura e do substrato na germinação de sementes de itaubarana (Acosmium nitens (Vog.) Yakovlev) - Leguminosae,

Caesalpinoidea. Acta Amazonica, v.35, n.1, p.35-39, 2005.

VZZOTTO, V. R.; MARCHEZAN, E.;

SEGABINAZZI, T. Efeito do pisoteio bovino em algumas propriedades físicas do solo de várzea. Ciência Rural, v.30, n.6, p.965-969, 2000.

ZAR, J. H. Bioestatistical analysis. 4.ed. Englewood Cliffs: Prentice Hall, 1999. 